\title{
Youssef Syndrome Following Vaginal Birth after Cesarean Section: A Case Report
}

\author{
${ }^{1}$ Vineet V Mishra, ${ }^{2}$ Ritu Agarwal, ${ }^{3}$ Sakshi Nanda, ${ }^{4}$ Sumesh Choudhary, ${ }^{5}$ Bhumika Vyas, ${ }^{6}$ Kavita Mistry
}

\begin{abstract}
Youssef syndrome which is also known as vesicouterine fistula is a very rare condition with an incidence of $1-4 \%$. It is an iatrogenic complication, the cesarean section being a common etiology while vaginal birth after cesarean section is uncommon. It may have delayed presentation ranging from weeks to years. It can be diagnosed with sonography, cystoscopy, magnetic resonance imaging, but hysteroscopy is more reliable. The definitive management is a surgical treatment only.
\end{abstract}

Keywords: Cesarean section, Cystoscopy, Hysteroscopy, Youssef syndrome.

How to cite this article: Mishra VV, Agarwal R, Nanda S, Choudhary S, Vyas B, Mistry K. Youssef Syndrome Following Vaginal Birth after Cesarean Section: A Case Report. J South Asian Feder Obst Gynae 2018;10(Suppl 2):432-434

Source of support: Nil

Conflict of interest: None

Date of received: 10 August 2016

Date of acceptance: 14 February 2018

Date of publication: July 2019

\section{INTRODUCTION}

Vesicouterine fistula is a communication between the bladder and the uterus. First such case was reported in 1908 by Knipe et al. It is one of the urogenital fistulas which is least common in occurrence with an incidence of only about $1-4 \%{ }^{1}$ Its main symptoms are cyclic hematuria, amenorrhoea, urinary incontinence, menouria, and recurrent UTI. The triad of menouria, amenorrhea, and urinary continence is known as Youssef syndrome. This syndrome was firstly described in the literature in 1938, however, the term was coined in 1957 by Youssef AM. ${ }^{2}$

\footnotetext{
${ }^{1}$ Professor and Head, ${ }^{2,5}$ Senior Resident, ${ }^{3}$ Clinical Fellow,

${ }^{4,6}$ Assistant Professor

${ }^{1}$ Department of Obstetrics and Gynaecology, Institute of Kidney Disease and Research Hospital, Ahmedabad, Gujarat, India

${ }^{2-6}$ Department of Obstetrics and Gynaecology, Institute of Kidney Disease and Research Centre-ITS, Ahmedabad, Gujarat, India

Corresponding Author: Vineet V Mishra, Professor and Head, Department of Obstetrics and Gynaecology, Institute of Kidney Disease and Research Hospital, Ahmedabad, Gujarat, India, e-mail: vineet.mishra.ikdrc@gmail.com
}

\section{CASE REPORT}

A 25-year-old female presented in the gynecology outpatient department (OPD) with complaints of hematuria during menses since 2 and half years. She had a history of lower segment cesarean section (LSCS) for breech delivery about 5 years back followed by vaginal birth after cesarean about 2.5 years. Since then, she complained of haematuria during menses, with no complaints of urine leak or dysuria or hematuria otherwise. Her menstrual history revealed regular, cyclical periods with moderate flow. No complaints of dysmenorrhea were present. Her urine analysis during the menstrual periods revealed 100-120 red blood cells (RBCs) in urine with few pus cells, $<50 \mathrm{mg} / \mathrm{dL}$ albumin, rest was normal. While during her dry days, her urine analysis was absolutely normal with no RBC. On per speculum examination, on day 12 of her menstrual cycle (menses absent), she had a normally situated urethral opening with normal healthy cervix and vagina, per vaginal examination revealed normal mobile parous sized uterus with normal free bilateral fornices. Her USG KUB was normal. Her routine pelvic sonography revealed, fistulous communication between the anterior uterine wall at the isthmic region with posterior bladder wall (Fig. 1). A probable diagnosis of Youssef syndrome was made. To confirm our diagnosis and before undertaking any surgical maneuver, we took the patient for cystoscopy and hysteroscopy. Cystoscopy was done with 17 french cystoscopes $\left(30^{\circ}, \mathrm{Karl}\right.$ Storz). It revealed a posterior wall opening of about $2 \times 2 \mathrm{~cm}$ at $3 \mathrm{~cm}$ above the interureteric ridge (Fig. 2). While negotiating through the opening, scope entered the uterine cavity at the level of isthmus. Uterine fundus with bilateral uterine ostia was

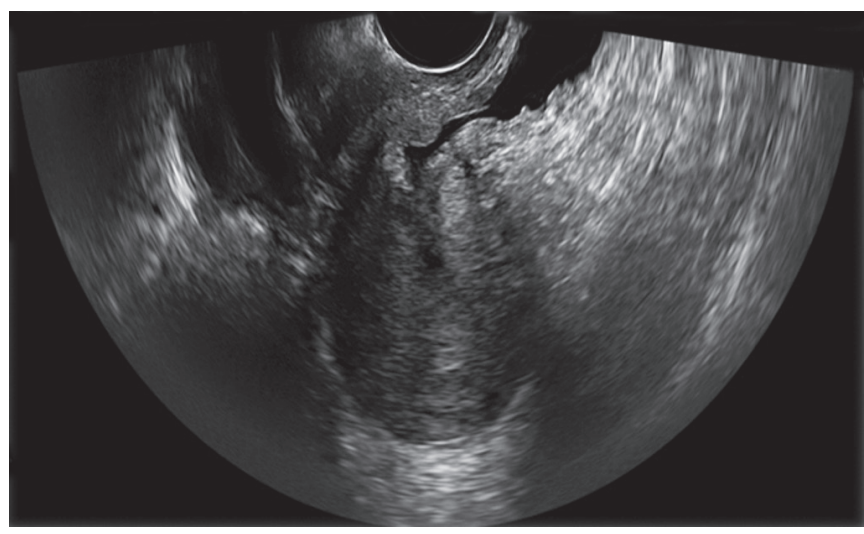

Fig. 1: Sonographic view of vesicouterine fistula 


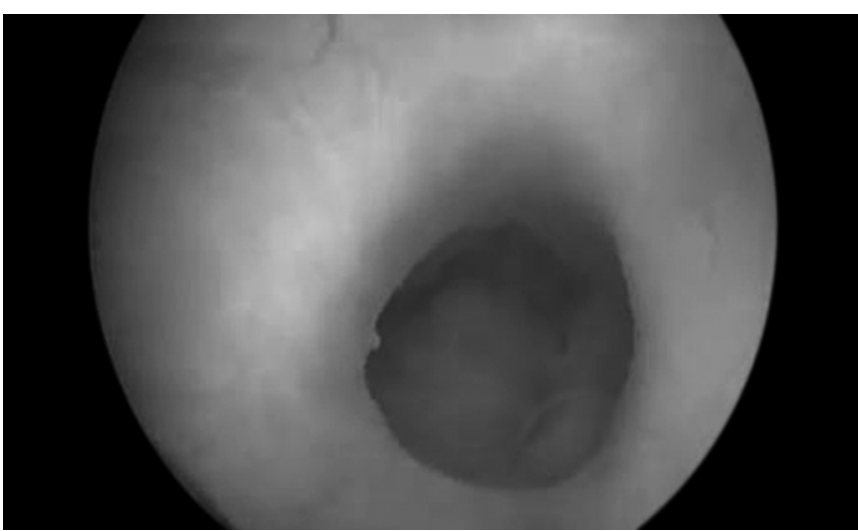

Fig. 2: Cystoscopic view of fistula

very well appreciated. Hysteroscopy was also performed in the same sitting, which confirmed the opening on the anterior uterine wall at the level of the isthmus. Definitive surgery was planned for the patient in the next OT and urine culture was advised to rule out any urinary tract infection prior to surgery.

On the day of surgery, patient painted and draped followed by self-retaining catheterization with 16 french foleys catheter. The abdomen was opened by Joel-Kohen incision deep up to peritoneal peritoneum. The uterus was exteriorized and held with Bonney's uterine holding clamp. Uterovaginal fold was identified and cut, sharp dissection done to separate the bladder from the uterus to reach the site of the fistula. The fistulous tract was excised with sharp dissection. The uterus was closed by interrupted sutures while bladder was watertight closed in 2 continuous interlocking layers. The omental fat pad was brought through one of the broad ligaments and interposition done between uterus and bladder at the site of fistula (Fig. 3). Suprapubic catheterization (SPC) was done to provide an alternative path for drainage of urine and to help in tension-free healing of bladder sutures. Per urethral catheter was removed on the next day and patient discharged with SPC in situ and advised follow-

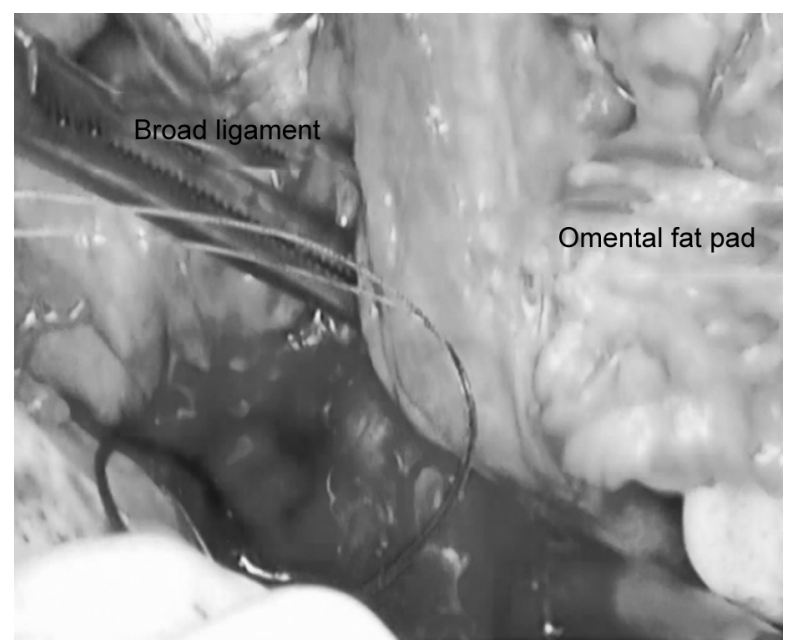

Fig. 3: Omental fat pad interposition between uterus and bladder up after 21 days. After 3 weeks, micturating cystogram was done to check for leak-free healing of bladder (Fig. 4). The repair site was found intact, and SPC removed. On 6 months follow-up, the patient is asymptomatic.

\section{DISCUSSION}

Youssef syndrome many a time is iatrogenic, most common cause being cesarean section ${ }^{3}$ followed by induced abortion, dilatation and curettage, vaginal birth after previous cesarean section, obstructed labor, forceps delivery, placenta percreta, migrated intrauterine contraceptive device, and brachytherapy. ${ }^{4}$ In our case, it was VBAC. Its most probable pathophysiology being pressure necrosis of bladder and uterine incision site due to prolonged labor. It is characterized by cyclic hematuria, amenorrhea, and urinary tract infection. Patients usually do not have a complaint of urinary incontinence because of functional cervical sphincter present below the level of fistula. But urinary incontinence can occur if the level of the VUF is at or below the level of internal os or if the os is incompetent. ${ }^{4}$ Most of these cases have delayed presentation ranging from a few weeks to years following the causative procedure. ${ }^{5}$ In our case, the patient was having symptoms 6 weeks following VBAC. However, there can be a considerable delay in diagnosis because examination classically used to depict the fistula may be negative. ${ }^{6}$ Hence a high level of suspicion is required for diagnosing this condition.

The fistula is often diagnosed by imaging studies and diagnostic cystoscopy. Park et al. demonstrated on USG the presence of fistulous tract as double echogenic lines between the anterior uterine wall and posterior bladder wall. ${ }^{3}$ Transvaginal sonography (TVS) can routinely be used as a diagnostic modality for VUF. ${ }^{6}$ In our case too, the fistula tract was very well visualized on TVS. However, hysterography was found to be the most reliable diagnostic tool by Tancer's review of published literature.4 MRI is the investigation of the first choice in diagnosing VUF, ${ }^{7,8}$

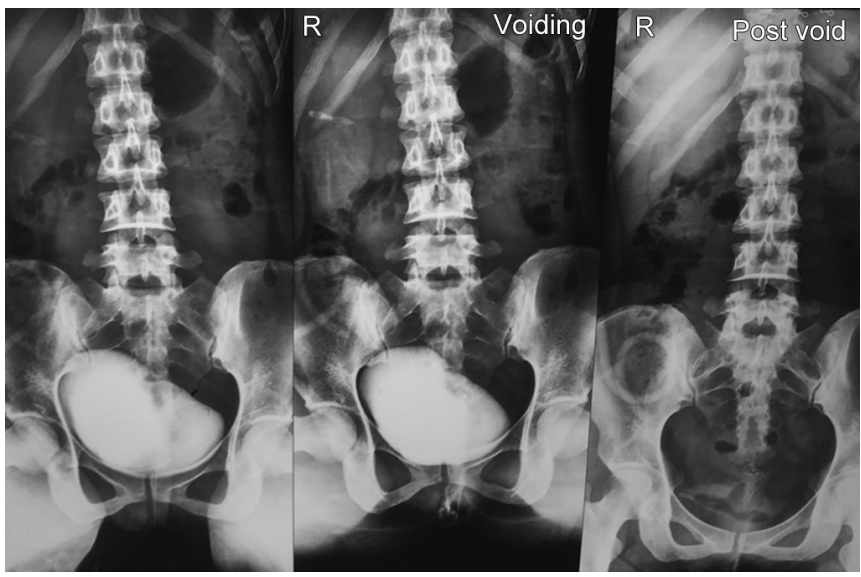

Fig. 4: Micturating cystourethrogram showing no leak of dye 
Treatment of VUF includes expectant, medical and surgical management. Expectant management, i.e., wait and watch has also proven successful as in some cases, it can cause spontaneous closure of fistula. ${ }^{9}$ Medical management involves induction and maintenance of prolonged amenorrhea with oral contraceptive pills, progestational agents and gonadotropin-releasing hormone analogs. ${ }^{10}$

Surgery is, however, the definitive treatment of choice. Initially, surgical treatment consisted of hysterectomy followed by the closure of bladder fistula opening. ${ }^{11}$ Because of advances in technology, this radical approach is being replaced by a conservative approach, i.e., preserving uterus and repair of the fistulous tract. It can be done transabdominally, transvaginally and laparoscopically. The transvaginal route is not a preferred route as fistula is higher in location and difficult to approach through the vagina. Whereas, the transabdominal repair is preferred. It can be done either extraperitoneal or retrovesical ( $\left.\mathrm{O}^{\prime} \mathrm{Connor}\right)$ technique. ${ }^{12}$ We preferred the retrovesical approach because of the surgeon's expertise in it. Increased morbidity, long hospital stay, and increased blood loss are the drawbacks of the transabdominal route, which can be overcome by laparoscopy approach. ${ }^{6}$ But laparoscopy approach requires surgical expertise and experience in intracorporeal suturing.

\section{CONCLUSION}

Although vesicouterine fistula is a rare condition, its incidence has risen due to an increase in cesarean rates and VBAC. Being an iatrogenic complication, it is difficult to prevent. However, by being vigilant, we can try to prevent, diagnose and manage it early, before it is too late for creating a socioeconomic and psychological burden to the female.

\section{REFERENCES}

1. Iloabachie GC, Njoku O. Vesico-uterine fistula. British Journal of Urology 1985;57(4):438-439.

2. Youssef AF. Menouria following lower segment cesarean section: a syndrome. American Journal of Obstetrics \&Gynecology 1957;73(4):759-767.

3. BK Park, SH Kim, et al. Vesicouterine fistula after cesarean section: ultrasonographic findings in two cases. Journal of Ultrasound in Medicine 1999;18(6):441-443.

4. Tancer Ml. Vesicouterine fistula-a review. Obstetrical and Gynecological Survey 1986;41(12)743-753.

5. Smayra T, Ghossain MA, et al. Vesicouterine fistulas: imaging findings in three cases. American Journal of Roentgenology 2005;184(10):139-142.

6. Perveen K, Gupta R, et al. Robotassisted laparoscopic repair of rare post-cesarean section vesicocervical and vesicouterine fistula: a case series of a novel technique. Urology 2012;80(2):477-482.

7. Murphy JM, Lee G, et al. Vesicouterine fistula: MRI diagnosis. European Radiology 1999;9(9):1876-1878.

8. Narayanan P, Nobbenhuis M, et al. Fistulas in malignant gynaecologic disease: etiology, imaging, and management. Radiographics 2009;29(4):1073-1083.

9. Brodhead GL. Spontaneous closure of large vesico-uterine fistula. Medication Reconciliation 1920;98:437.

10. Yip SK, Leung TY. Vesicouterine fistula: an updated review. International Urogynecology Journal and Pelvic Floor Dysfunction 1998;9(5)252-256.

11. Netto NR Jr, Santos PQ . Youssef's syndrome. Int Urol Nephrol 1975;7(2):115-118.

12. Dalela D, Ranjan $P$, et al. Supratrigonal VVF repair by modified O'connor's technique: an experience of 26 cases. European Urology 2006;149(3):551-556. 